\title{
Invisible Cause of Death in Global Road Safety \\ Crisis: A New Paradigm
}

\section{Short title: Driving with a clear field of vision can help save lives}

\author{
Nirmalchandra. K Shetty \\ Shri Krishna Research Centre, Mumbai, India.

\section{Corresponding Author:} \\ Nirmalchandra K Shetty \\ E-mail: shrikrishna.rc@gmail.com
}

Article category: Research Article

Preprint DOI: $10.31219 / 0 s f . i o / d y 4 v h$

\begin{abstract}
Global road safety is a complex problem. Annually 1.4 million people are killed, while over 50 million are injured in road traffic crashes worldwide. It endangers the victims' life or health and has a heavy social and economic impact on their families, medical facilities, and the nation's economy.

There have been numerous global efforts by the UN, WHO, World Bank, among others, to address the road safety crisis. According to research, human error is the root cause of $90 \%$ of crashes, which is largely attributed to the driver. In 2006, a serendipitous idea led to my discovery of the invisible primary cause of road traffic deaths and injuries - the obstruction of the driver's field of vision by the blind region of the A-pillar in all vehicles, which is a universal design flaw. Road traffic crashes are difficult to avert if vehicles and other road users or objects are invisible to the driver before the crash. Thus, this problem, including its proposed A1-pillar design solution, was conveyed to the UN, WHO, among others, in 2006. In spite of the positive response, these organisations did not adopt it as part of global road safety initiatives.

Despite commendable efforts by global institutions and governments, stricter traffic laws, and automotive safety technology advancements, road traffic deaths and injuries are steadily increasing. Could a key causative factor have been ignored so far? Thus, this article explores the blind region created by the A-pillar and offers a sustainable design solution for new vehicles as well as a retrofit idea for existing ones. Urgent action is needed to validate and overcome this major causative factor in road traffic deaths and injuries worldwide. Along with the existing global road safety measures, this comprehensive solution has the potential to help resolve the global road safety crisis and save lives.
\end{abstract}

Keywords: Road safety, road traffic deaths, injuries, save lives, driver, vehicle design, sustainable developmental goals. 


\section{Introduction}

\section{Magnitude of the Global Road Safety Crisis}

The rate of road traffic crash (RTC) deaths and injuries globally every year has been alarming. From 1.26 million deaths in 2010, it increased to 1.35 million in 2016; this means that 3,800 people are killed each day, and one person dies every 24 seconds. Moreover, there are over 50 million people who are seriously injured annually. According to a previous study, 93\% of road traffic deaths and injuries occur in low and middle-income countries (LMIC) [1], with over 50\% of deaths involving vulnerable road users (i.e., pedestrians, cyclists, and motorcyclists). In fact, it is the largest cause of death among children and young adults aged 5-29 years [2]. The socio-economic burden it brings to families, communities, and nations is colossal. It is estimated that the global economic loss due to RTC deaths and injuries is about $3-5 \%$ of the global GDP [3], which is likely to have increased substantially from USD 518 billion in 2005 [4]. It costs LMIC over 6.5 percent of GDP or roughly 1.7 trillion dollars every year [5].

Table 1. The magnitude of global road safety crisis

\section{Global Road Safety Crisis - Analysis (Based on WHO \& World Bank estimates)}

1. Total No. of people killed in 2006

2. Total No. of people killed in 2018

3. Total No. of people injured annually

4. Total No. of people killed in Low \& middle income countries (93\%)

5. Total No of people killed in Developed countries (7\%)

6. No. of people killed in a day (including one child every 3 minutes)

7. Percentage of pedestrians, cyclists deaths

8. Motorised 2-3 wheelers deaths

9. Percentage of car occupants deaths

10. Unidentified road user deaths

11. Leading cause of death in children age group

12. Total cost to GDP of most countries
(Approx.)

1.26 million

1.4 million

$>50.0$ million

1.3 million

0.1 million

3800

$26 \%$

$28 \%$

$29 \%$

$17 \%$

$5-29$ years

$3-5 \%$

Many families in LMICs instantly fall below the poverty line if the victim is the sole earning member of the family. Life is too precious to be lost or injured due to road traffic crashes.

Over the decades, the United Nations, World Health Organisation, World Bank, United Nations Road Safety Council (UNRSC), and United Nations Economic Commission for Europe (UNECE), among others, with the support of stakeholders like governments, vehicle manufacturers, roadway authorities, academic research institutes, and NGOs, have undertaken numerous initiatives to improve road safety.

Some of the key causative factors that they have addressed are speeding, child restraint, distracted and drunk driving, and the use of seatbelts and motorcycle helmets. In addition, they also focused on vehicle safety standards, road infrastructure, traffic signals, traffic lane markings, and 
emergency trauma care, among others. However, despite these concerted efforts, the target of the UN's Decade of Action for Road Safety 2011-2020 [6] and Sustainable Development Goals (SDG) 3.6 [7], which is $50 \%$ reduction of road traffic crash deaths and injuries by 2020 , could not be achieved. On the contrary, road deaths and injuries continued to increase during that period.

The United Nations subsequently declared 2021-2030 as the $2^{\text {nd }}$ Decade of Action for Road Safety, with a similar target of 50\% reduction in road traffic deaths and injuries by 2030 [8]. However, the increasing trend in RTC deaths and injuries indicates the possibility of a causative factor that has gone largely unnoticed.

\section{Analysis of the Problem Areas in Global Road Safety}

The following are two key statistics from WHO and World Bank reports:

1. $93 \%$ of RTC deaths occur in low and middle-income countries (LMIC), and

2. $90 \%$ of RTC deaths and injuries are caused by human error [9].

These factors are paramount to identifying the core problem and creating a solution to have a sizeable impact on Global Road Safety Crisis. In addition to the existing global road safety measures, new innovative breakthrough solutions are required.

To conceptualise an effective road safety strategy based on data analysis, there is a need to differentiate road safety issues at a fundamental level into:

1. Factors that help prevent RTC, and

2. Factors that help minimise post-crash effects on victims.

Prevention of RTC is of prime importance. Success in the prevention of RTC will automatically reduce the need for post-crash safety measures.

\section{Human Error - cause of $90 \%$ of road traffic deaths}

Global data shows that $90 \%$ of RTC is due to human error [9], which is largely attributed to the driver's fault. Some major factors that drivers should avoid, to help prevent RTC are breaking traffic rules, speeding, driving while drunk or under the influence of drugs, distracted driving, using a mobile phone and unsafe vehicles, reckless driving, bad roads, overloading, and driving under conditions that affect the ability to drive, such as drowsiness, fatigue, eyesight issues, and health problems. These factors can be addressed through awareness, training, and enforcement. However, the risk of RTC due to any of the above factors is magnified if the driver's field of vision is also hampered.

Moreover, the major factors that help minimise post-crash effects are the use of seat belts, child restraints, air bags, helmets, crumple zones, and emergency medical services.

Despite global preventive and post-crash efforts, the total number of road deaths and injuries, especially in LMIC and some developed countries like the USA, has increased. This indicates the strong possibility of a potential causative factor that has not been previously addressed. Thus, identifying the 
potential missing factor and addressing it could lead to a significant reduction in global RTC deaths and injuries.

\section{Methodology}

To have any sizeable impact on global road safety, our research focused on the causative factor responsible for $90 \%$ of RTC-human error (i.e., the driver). Thus, it is important to ascertain the most crucial factor that would help drivers in the prevention of human error-a clear field of vision.

One of the essential attributes for safe driving is that the driver must have a clear field of vision to perceive danger ahead of time and respond appropriately to avert the RTC by manoeuvring the vehicle and slowing down or braking abruptly, depending on the situation.

\section{The blind region caused by the A-pillar-a universal flaw in vehicle design}

A major potential cause of RTC deaths and injuries is a 'universal flaw in vehicle design-the blind region caused by the vehicle's A-pillar', which is a critical area in the field of vision of the driver. Although crucial, this has not been addressed by international organisations and vehicle manufacturers. It has a direct impact on road safety, as it partially blocks the driver's field of vision. At certain points, the driver will be unaware of other road users, traffic signals, and road signs, among others, which fall in the blind region of the A-pillar. Thus, the blind region of the A-pillar must not fall within the driver's field of vision or the trajectory of the vehicle.

A crucial factor in driving is to be able to see the road clearly. Over $80 \%$ of the decisions taken to avoid RTC are based on good vision, since it enables the driver to make timely decisions to avert RTC. If the driver is not able to see other road users, objects, or traffic lights, there is little probability of preventing the crash. The obstruction caused by the blind region of the A-pillar has been depicted in Figure 1. The blind region varies between different types and models of vehicles based on the design, size of the A-pillar, and distance from the eyes of the driver to the A-pillar and the object.

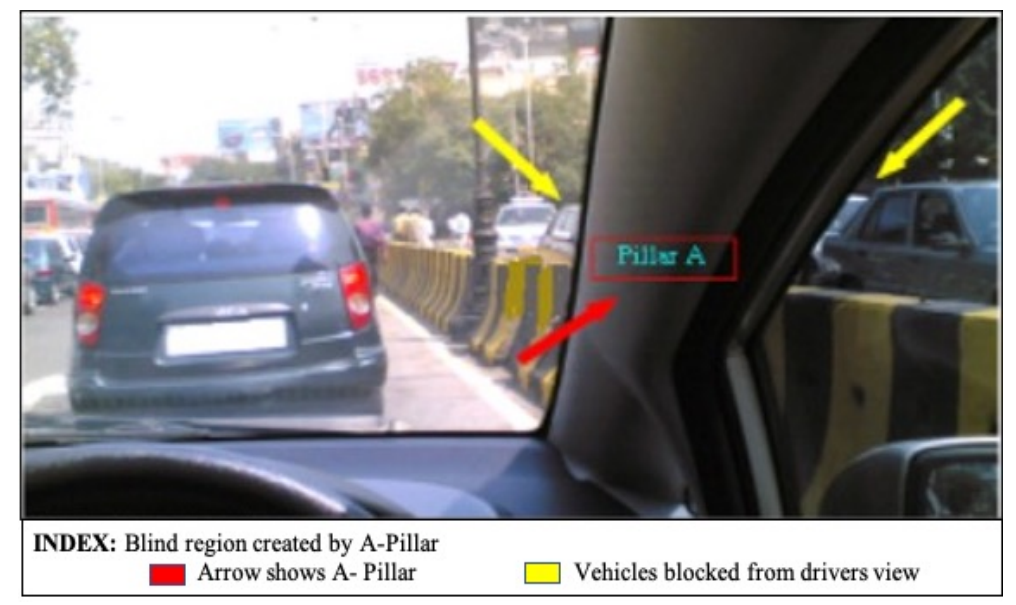

Figure 1. The blind region caused by the A-Pillar is depicted. Vehicles and vulnerable road users on the other side of road divider are partially blocked from the driver's view by the A-Pillar. 


\section{How the blind region impacts safe driving (Figure 2)}

To drive safely and avert RTC, the driver must have a clear view of the road. However, due to the blind region caused by the A-pillar, a section of the field of vision is blocked. The blind region caused by the A-pillar on the driver side is broader than on the passenger side because of proximity to the driver's eye. It widens as the distance from the vehicle's A-pillar increases from the driver's point of reference.

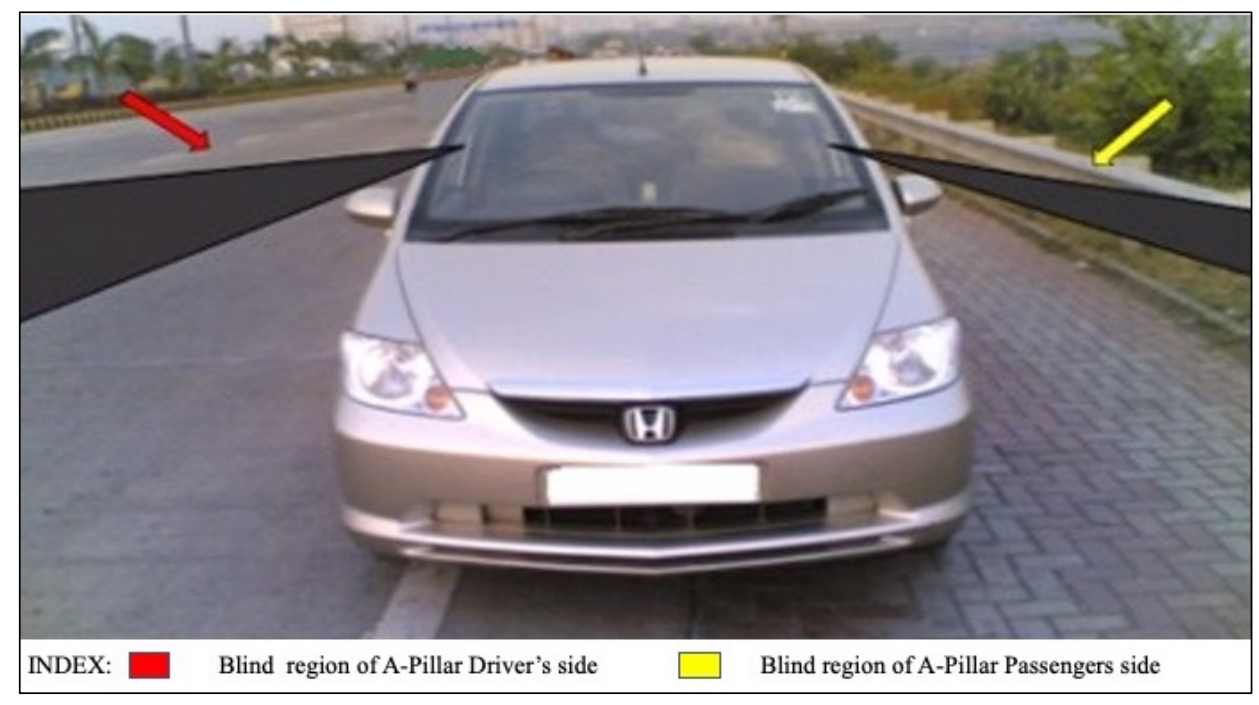

Figure 2. The blind regions caused by both A-pillars.

Road traffic (e.g., pedestrians, cyclists, motorcyclists, cars, vans, buses, and trucks), entire lanes of traffic at crossroads, pedestrian crossings, traffic lights, and road traffic signs, as well as vehicles approaching from narrow roads merging on to the main road, can be blocked from the driver's view by the A-pillar.

Averting RTC depends, to a great extent, on the quick response of the driver. It only takes a fraction of a second for a crash to occur. Moreover, there is a lag time between the perception of the danger ahead and response to brake, as well as the distance the vehicle travels before coming to a halt, based on the vehicle type and speed. However, if the driver is oblivious to the danger ahead due to a blind region, there might be a delayed or even no reaction to avoid the crash. Thus, the blind region in the driver's field of vision is a major causative factor for RTC.

As the vehicle moves, the blind region blocks part of driver's view progressively. The driver may be aware of some objects being blocked by the A-pillar, especially when travelling along a straight road paying full attention. However, it is possible for objects to fall within the blind region without the driver realising it because of momentary diversion of attention or distracted driving. In a short span of time, a vehicle moving at $50 \mathrm{~km} / \mathrm{h}$ could have covered a distance of about 90 feet in 2 seconds. Thus, a driver can easily miss traffic signals, other vulnerable road users, or vehicles at intersections.

For example, if a car follows the trajectory of the blind region caused by the A-pillar, and other vehicles or vulnerable road users ahead are in the direction of its moving blind region trajectory, the probability of RTC is increased (Figure 3). 


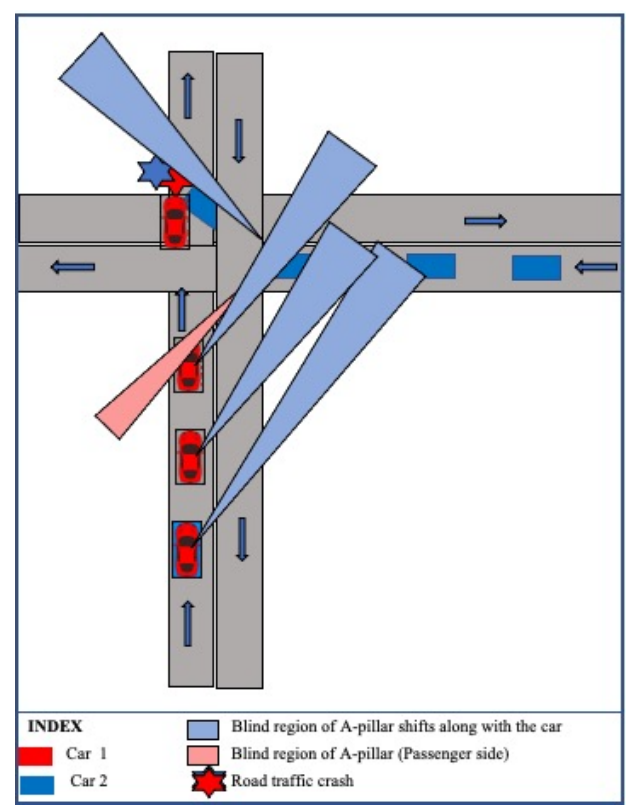

Figure 3. Driver's field of vision blocked by blind region of A-Pillar in right hand drive cars.

In this study, we tried to evaluate the extent to which the blind region of A-pillar blocks the field of vision of the driver, including its effects and the probable solution to overcome it. Through an improved field of vision, the driver can constantly receive complete real-time information about the surroundings and take more life-saving decisions in a timely manner.

In 2006, vide our letter on 'Global Road Safety Crisis-Visionary Solutions (serendipity), this universal flaw in vehicle design, as well as the solution to overcome it, was brought to the attention of the UN, WHO, World Bank, UNECE, UNRSC, and vehicle manufacturers, among others.

Unfortunately, this problem is still unaddressed.

\section{Solution for blocked field of vision caused by the A-pillar :modifications in car (Figure 4)}

To overcome the blocked field of vision caused by the A-pillar, a slight modification in the basic design

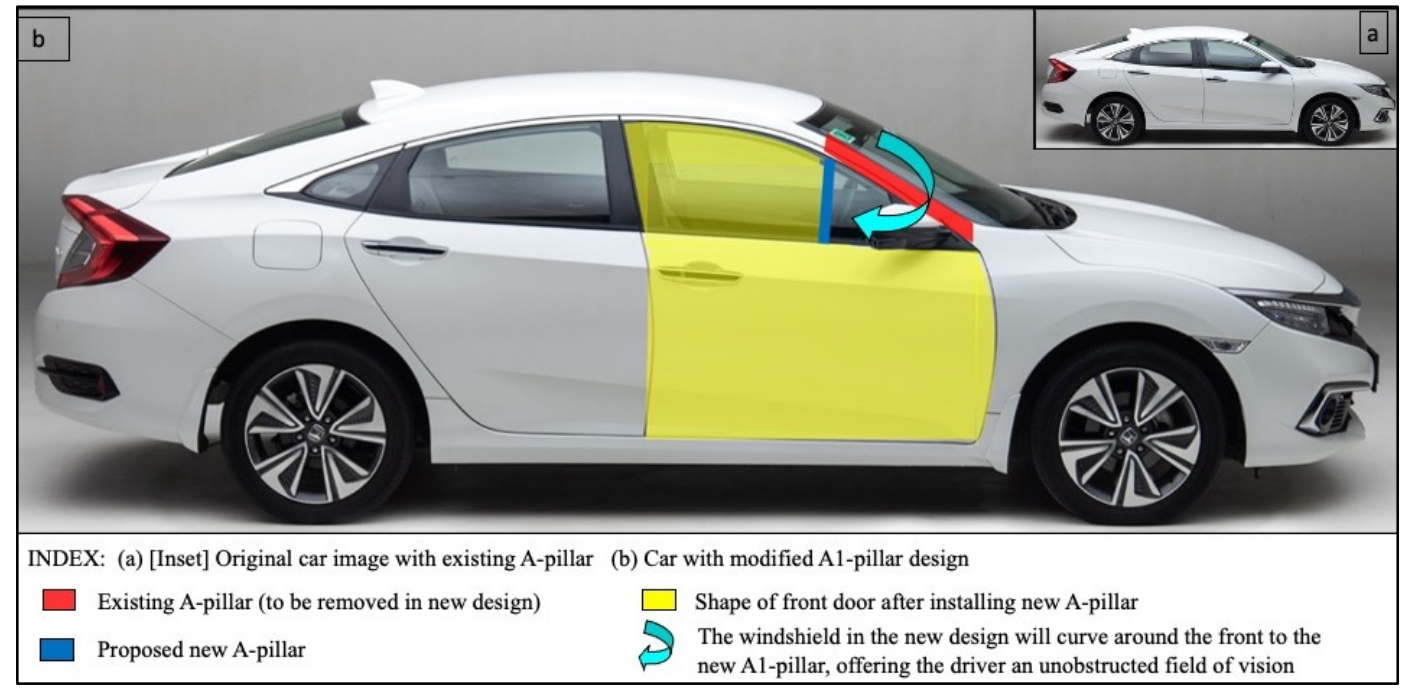

Figure 4. Model of Car with proposed A1-Pillar - Modifications to address Blind region of A-Pillar a) Original model of car. b) Proposed modification of car design with A1-Pillar. 
(concept) of the car needs to be carried out in both A-pillars, as follows:

- The A-pillar should be shifted to a new position called the A1-pillar, located between the backrest of the driver's seat and the steering wheel, as viewed from the side of the car. It is ensured that shifting the position of the A-pillar to the A1-pillar does not cause any obstruction for easy entry and exit of the driver (Figure 5).

- The windshield should be extended to curve in the region of the existing A-pillar along the door, up to the new A1-pillar. Thus, the width of the new A1-pillar should be as narrow as possible.

- The structural support for the extended windshield and the new A1-pillar has to be provided.

- The size of the window should be reduced slightly to house the extended windshield and A1pillar.

- The shape of the front door should be modified partially to accommodate the new curved windshield and A1-pillar. The front door opens from under the A1-pillar and the extended windshield.

Our tests showed that an average-built adult could effortlessly climb in and out of the test car. Even in existing vehicles, the space above the lower end of the steering wheel is not used when entering or exiting the vehicle.

\section{Model of the car with modifications to the A-pillar and door (Figure 5)}

The modification suggested above was carried out in a Honda City car.

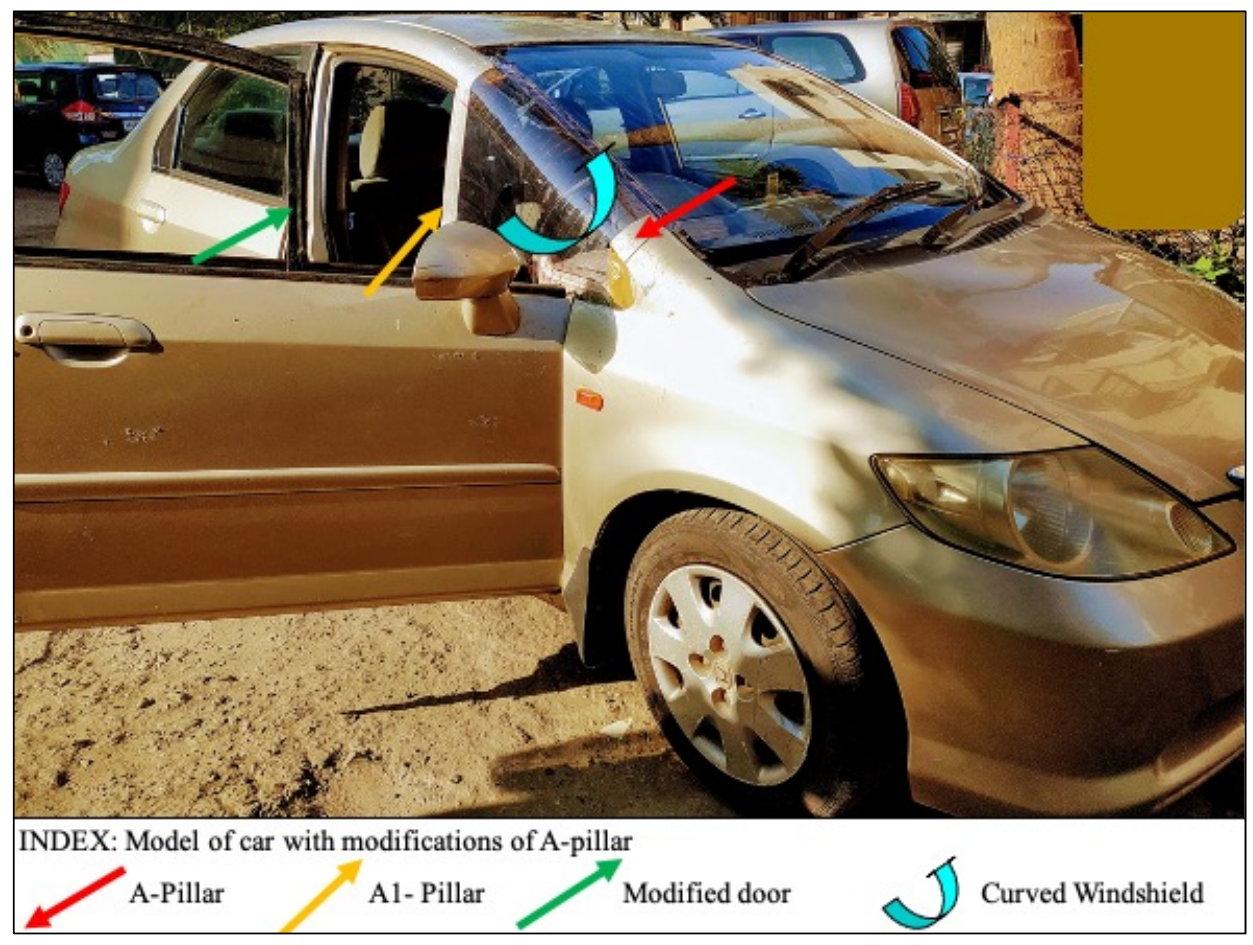

Figure 5. Modified car - changes made in driver's side of the car a) A-pillar removed b) Position of new A1-pillar c) Modified door to accommodate the curved windshield 
- The A-pillar on the driver's side was cut, except for small sections at the top and bottom of the pillar, which were retained to indicate its original position.

- The new A1-pillar was narrower than the original; it was installed on an extended steel arm from the A-Pillar base to the level aligned to the front lower end of the steering wheel.

- The windshield was extended to the A1-pillar using transparent PVC sheet.

- A section of the extended windshield represents the region covered by the original A-pillar.

- The extended windshield up to the A1-pillar gives a wide unobstructed field of vision for the driver in the modified car.

- The door was also modified to accommodate the extended windshield up to the A1-pillar.

- The door opens freely, and the driver can easily enter and exit the car.

Subsequently, the car was tested on the road to ascertain if the new modified A1-pillar offered any differences or advantages, as compared to the existing A-pillar. If two cars were used to carry out the road test -one with the existing A-pillar and the other with the modified A1-pillar; the road traffic scenario would have been different for both, and thus difficult to assess as regards differences and advantages. Therefore, we decided to use only one car; we clearly defined the blind region of the existing A-pillar (which was removed) using a red shaded transparent area with cross lines, which was located on the curved windshield that extends to the modified A1-pillar. Photographs and a video recording were taken using a smartphone placed near the eye-level of the driver to give a realistic perspective of the driver's field of vision.

\section{Retrofitting of existing vehicles to overcome the A-pillar problem (Figure 6)}

The proposed concept of the new A1-pillar, along with a curved windshield and new door design, is applicable for new vehicles to provide drivers with an unobstructed view. As for existing vehicles, shifting the A-pillar to the A1-pillar is expensive, unsafe, and might not meet vehicle safety requirements. However, in order to help resolve the global road safety crisis, existing cars (over 1.4 billion) and millions of other large vehicles would need to be retrofitted to overcome the A-pillar problem.

In existing cars, the problem can be solved by installing a wide angle front-view dashcam on the external surface of the roof of the vehicle, with the display screen placed on the dashboard in front of the driver. The placement of the dashcam is important. The forward view dashcam ideally needs to be placed at a position above the driver's head, close to an imaginary line that might join the B-pillars, and adjusted to offer a good field of reference to the driver (Figure 6). This position will cover the region blocked by the A-pillar, which is not the case if the dashcam is attached to the windscreen or rear-view mirror. 


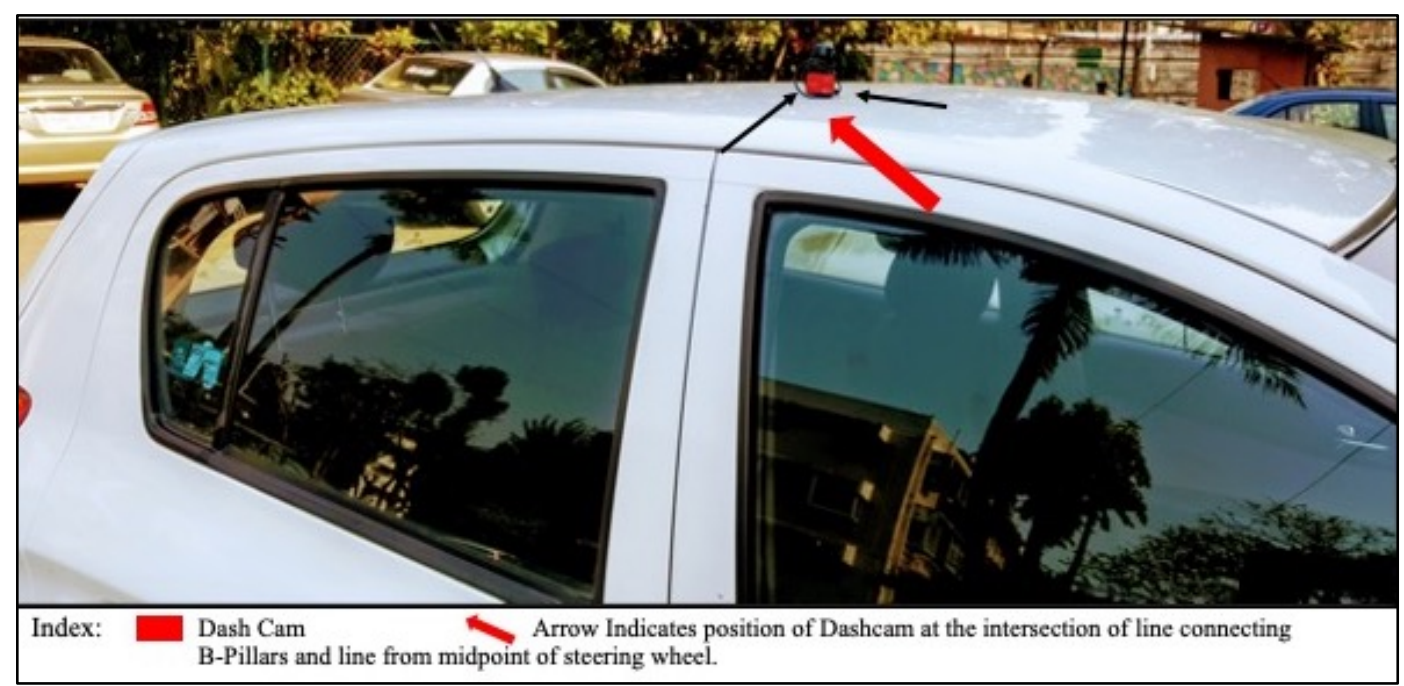

Figure 6. Retrofit Dashcam - to overcome the bind region of A-Pillar. Placement is atop the car. Aligned directly above the driver's head.

Photos that were simultaneously taken at a signal light from inside the vehicle and atop the car are shown in Figure 7. The difference between the photos (Figures $7 \mathrm{a}$ and $7 \mathrm{~b}$ ) shows the extent of road information that does not reach the driver; it indicates how shifting the A-pillar to the position of the
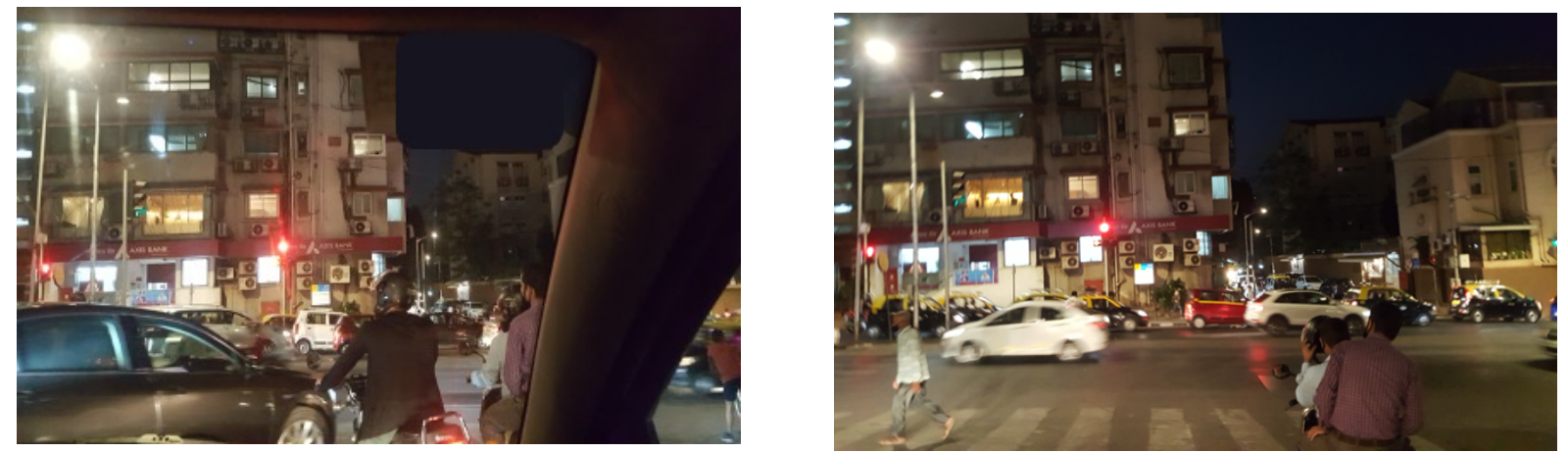

Figures $7 \mathbf{a}$ and $7 \mathbf{b}$. Depiction of the objects blocked by blind region of A-pillar. The two photos were taken at the same spot at a traffic signal. Figure 7a depicts the driver's view from inside the car. Figure $7 \mathrm{~b}$ taken from the top of the car above the driver's head, clearly depicts the objects blocked by the blind region caused by the A-pillar.

A1-pillar in new vehicles or using a retrofit dashcam in existing vehicles may enable safe driving as it gives a wider view of the traffic, including objects that are blocked by the A-pillar.

The modifications above would be applied to all new motorised vehicles (excluding two wheelers). The model of a truck with a proposed A1-Pillar is shown (Figure 8).

- Large side view mirrors on trucks and buses, given their position right next to the A-pillar, tend to widen the blind region. The mirrors should ideally be placed at a higher position, so as to serve the purpose of a side view mirror, without obstructing the driver's forward field of vision.

- Adding a glass pane in the front bottom section on both sides of all heavy vehicles, ahead of the doors, will give the driver a better view of children and objects near the front tyres. It would also enable the driver to obtain a view of the edge of the road, especially when driving on narrow roads or close to the edge on mountainous roads. This could help prevent buses or trucks from accidentally falling down the valley. 

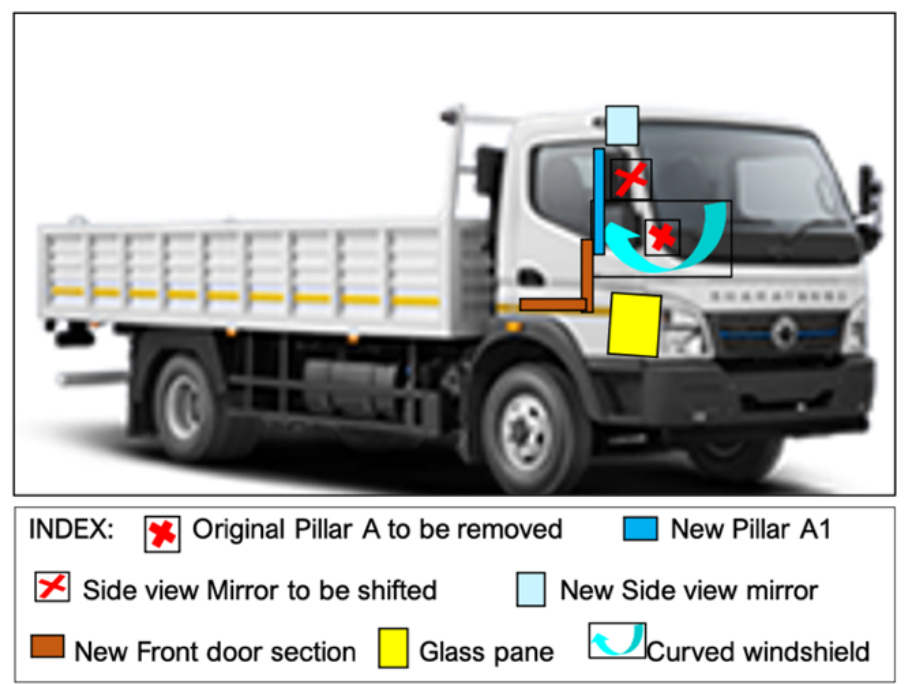

Figure 8. The Model of truck with proposed A-1 pillar

\section{Results}

A photo taken during the road test of the modified car depicts the road traffic scenario (Figure 9). As the A-pillar has been removed from the driver's side of the car, the red striped transparent section measuring the width of the original A-pillar serves as a representation of the A-pillar and shows the region that is blocked from the driver's field of vision. The red striped transparent section depicts an entire lane of traffic blocked from the driver's view.

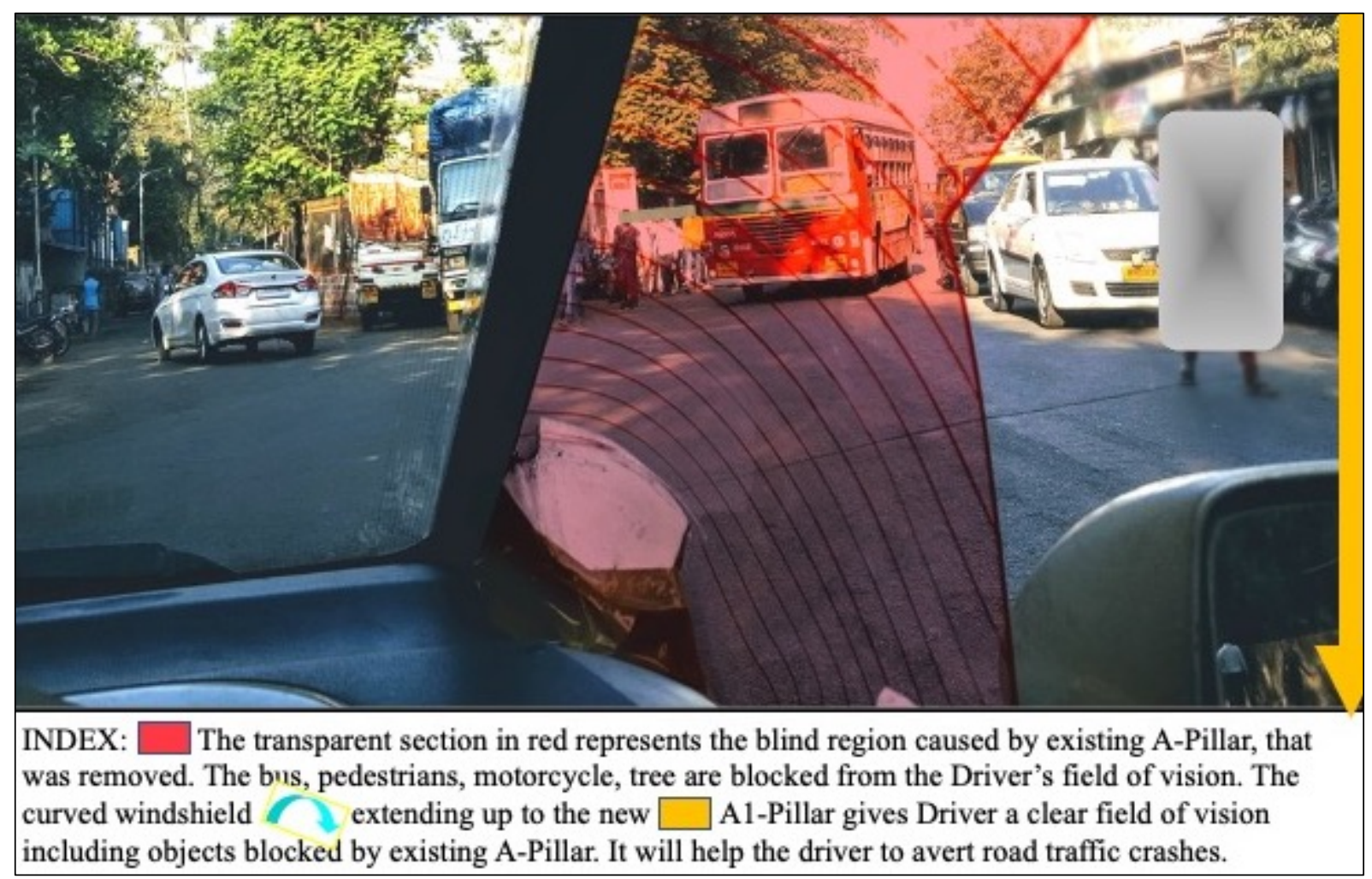

Figure 9. Photo of a road traffic scene. a) Existing A-Pillar: Crucial images blocked from Drivers view. b) A1Pillar: The curved windshield provides driver a clear wider field of vision (including images blocked by A-Pillar) 
The driver's field of vision with the existing A-pillar and the modified A1-pillar could be assessed simultaneously from images of any road traffic situation, as the difference could be noticed instantly.

\section{Assessment of existing A-pillar and modified A1-pillar}

Existing A-pillar (red striped transparent section): The photo gives a clear view of a bus, motorcycle, partial truck, car, and pedestrians that are blocked by the blind region of the A-pillar. This shows that valuable information about the road traffic, including road signs and traffic signals is blocked from the driver's view. Being unaware of the traffic behind the A-pillar could affect the ability of the driver to avert or accidentally cause RTC.

New modified A1-pillar: This gives the driver access to a wider unobstructed field of vision of the same traffic up to the A1-pillar, including the traffic covered in the red striped section that is blocked by the original A-pillar. Moreover, it gives the driver the advantage to take timely action.

The black band on the existing windshield called frit, which is used for bonding the windshield to the frame of the vehicle, is normally concealed by the A-Pillar. With the proposed A1-pillar design for future vehicles, the frit of the curved windshield shall be moved to the A1- pillar position, thus improving the field of vision further.

It is clear that drivers can only prevent a road crash if they can see the risk of a possible crash in time. By driving around different roads and intersections, it became increasingly evident that the current design of the A-pillar in all vehicles could potentially be a major causative factor for road traffic deaths and injuries worldwide. Drivers would be oblivious to situations or risks behind the Apillar, unless they look on either side of the A-pillar during critical times, at intersections, and while turning or changing lanes.

This comprehensive solution offered to overcome the blind region caused by A-pillar by utilising a modified A1- pillar design for new vehicles and a retrofit dashcam for existing vehicles could help in improving the field of vision of drivers and reduce global road traffic deaths and injuries considerably.

\section{Discussion}

A major causative factor for RTC is the 'blind region in the field of vision' of the driver. The A-pillar obstructing the field of vision of the driver is a crucial universal design flaw across all vehicles. However, it has not been addressed by global road safety initiatives so far.

- The invisible cause of road traffic deaths and injuries is the A-pillar. This blocks the driver's visibility of an entire lane of traffic, pedestrians, cyclists, motorcyclists, buses, trucks, and stationary objects like signal lights, road signs, and trees (Figure 9). Having a clear field of vision for drivers is an absolute necessity for safe driving. Being aware of the traffic behind the 
A-pillar could help drivers take timely action to prevent road accidents and avert RTC. Every microsecond that the driver is unaware of his surroundings causes a delay in responding and averting any potential danger. The A-pillar blocks out a section of their field of vision, which is further compounded with speed, distance, and distracted driving; these factors affect the driver's ability to react on time.

- At a distance of 3 metres from the vehicle, the blocked region is about 1 metre in width; at 20 metres, the blocked region is around 5 metres wide. Any vehicle or pedestrian falling inside the triangular blind region, which extends from the driver's eye position and the pillar, would not be visible to the driver (Figure 2). A driver can respond in time to avert a potential road mishap if the person or object is visible at a distance.

- In many cases of RTC, despite following every possible precaution and traffic rules, the driver is often taken by surprise at the sudden appearance of a pedestrian, cyclist, or any other vehicle or object in front of the vehicle. Many drivers are certain that they did not see the obstacle in time (possibly due to the invisible factor-the A-pillar) and their subsequent efforts to avert the RTC were unsuccessful, resulting in injuries or death of the victims on the road or the occupants of vehicles. Even after the brakes are applied, the vehicle moves some distance before coming to a halt.

- For example, if a car at a crossroads takes a 90-degree turn, and at a distance of 20 metres, a pedestrian crosses the road ( 5 metres wide) and falls in the trajectory of the blind region of the A-pillar ( 5 metres wide), the driver is not likely to avert a crash. This is because to cover 20 metres at a speed of $30 \mathrm{~km} / \mathrm{h}$, it will take 2.4 seconds, whereas the pedestrian requires over 4 seconds to cover the 5 metres to cross the road. The alertness and precaution taken by the pedestrian or the driver looking on either side of the A-pillar may prevent the RTC from taking place. Timely action to avert the crash can be taken by the driver if the A-pillar does not block the view.

- In an RTC between two vehicles that are driven cautiously, the blind region of the A-pillar in any or both the vehicles could obstruct the field of vision of the drivers, resulting in accidentally causing the crash or limiting their ability to avert it. The blind region becomes more problematic with bends in roads and intersections. While overtaking any vehicle, especially a heavy vehicle on two-way roads or highways, the driver of the car may align the side of the car with the vehicle ahead and try to look in front for oncoming vehicles. In the process, the A-pillar may block the driver's forward vision to some extent, resulting in RTC.

- All road users, including pedestrians, cyclists, and motorcyclists, also have a responsibility to keep the roads safe by observing traffic rules and being alert at all times. They may not be visible to drivers because of this invisible factor of the blind region of the A-pillar; thus, they should not rely on drivers for their safety. Sometimes, crashes are averted by the presence of mind of one or more parties in a potential crash. 
- A change to the design of the A-pillar across all vehicles would be an effective way of saving lives. Given the significant role of the A-pillar in delaying the driver's response time, many road crashes might not be due to the driver's fault alone, but must be shared by vehicle manufacturers as well because of faulty design.

\section{Cause for greater incidence of road traffic crashes in LMIC}

- The high incidence of RTC in LMIC may be attributed to several factors, such as dense population, poor road infrastructure, inadequate road safety management and traffic law enforcement, improper training for drivers, speeding, drunk or distracted driving, and lack of awareness of road safety among road users, besides others. These factors are magnified when the driver's field of vision is blocked by the A-pillar. The driver's response time and distance to come to a halt may be reduced considerably because of the dense population, meandering narrow roads, roads with sharp turns, narrow roads without footpaths, or the sheer proximity of other road users.

- Another factor affecting the driver's vision at night, especially in LMIC, is moving or stationary vehicles, especially heavy vehicles without working headlights, taillights, and lights on the sides of the vehicle. Without proper lights, these vehicles are often not visible on ill-lit roads.

\section{Manufacturer's role of incorporating safety provisions in vehicles}

- Manufacturers usually focus on making vehicles safe to minimize the effects of road crashes on their occupants. The result has been major changes brought about by advances in technology, including braking systems, crumple zones, seat belts, air bags, and automatic self-driven vehicles, among others. Besides, manufacturers should address other factors, such as:

- The A-pillar: A major factor in prevention of RTC, which is overlooked by the manufacturers, is the hindrance caused by the 'blind region of the A-pillar'. Urgent attention is required to rectify this major design problem of the A-pillar in all vehicles.

- Vehicle manufacturers must primarily focus on designs that will help prevent RTC to protect not only the occupants of the vehicle, but other vulnerable road users as well. The provision of a wide range of touch screen gadgets, such as a music and video player on the dashboard, indirectly promotes distracted driving. Sport utility vehicles (SUV) can cause greater risk to vulnerable road users because of the large size and shape compared to other cars.

- Side view Mirrors: Another flaw in vehicle design which misleads the driver is convex sideview mirrors in vehicles with the inscription 'objects in the mirror are closer than they appear'. It gives the driver the illusion that the approaching vehicle is at a greater distance than its actual position. This can result in a judgment error when changing lanes or making a turn if one depends on the side-view mirror. This avoidable judgment error may be the cause of many RTC deaths and injuries. Thus, vehicle manufacturers should avoid fitting convex mirrors in new 
vehicles. Retrofitting existing vehicles with side mirrors that are similar to those used in rearview mirrors may help reduce RTC to some extent.

- Speed: Another major factor related to RTC is over-speeding. Speed limits indicate the maximum permissible speed. However, it is advisable for drivers to resort to an appropriate speed that may be much lower than the speed limit to promote safe driving, depending on the country, city or town, road and traffic density, quality of roads, and density of vulnerable road users, among other factors. It is human tendency to over-speed, often beyond the limits. Thus, manufacturers of vehicle and motorcycles must lower the top speed limit of vehicles/motorcycles to prevent over-speeding and install active intelligent speed adaptation systems to help drivers adhere to speed limits.

\section{Conclusion}

The magnitude of the perennial problem of global RTC deaths (1.4 million) and injuries (over 50 million) annually is alarming. The concerted global efforts during the last two decades to reduce this problem by $50 \%$ by 2020 did not materialise; on the contrary, it increased. This clearly indicates that new innovative breakthrough solutions are required to bring about a drastic reduction in road traffic deaths and injuries globally.

Our research identified the invisible cause of road deaths and injuries globally, viz. the hindrance to the driver's field of vision caused by the blind region of the A-pillar, which is a universal design flaw. It is a crucial factor that has not been addressed in road safety initiatives so far by the international organisations or vehicle manufacturers. According to available data, $90 \%$ of RTC are due to human error that is largely attributed to the driver's fault. The traffic behind the A-pillar may be invisible to the driver, thus resulting in accidents or non-aversion of RTC.

We conceptualised the indicative design solution by shifting the position of the existing A-pillar to the new A1-pillar for new vehicles. A retrofit solution to overcome this problem is proposed for all existing vehicles. Recently, the proposed modifications were carried out in a car, viz., shifting the Apillar to the A1-pillar, and extending the windscreen to curve up to the A1-pillar, without causing any difficulty for the driver to enter or exit. This car was tested on the road; photos and videos were taken to capture the gravity of the problem alongside the solution. This clearly depicts the extent to which the Apillar blocks the crucial field of vision of the driver, and thus affecting the driver's timely response to avert the RTC. The clear, extended field of vision provided by the proposed A1-pillar offers valuable information to the driver to be more aware of the traffic for safe driving.

These concepts need to be tested and implemented on an urgent basis globally to make roads safe for all users, as the blind region of the A-Pillar may be the major causative factor of RTC especially in LMIC. Further research is encouraged to find ways of designing vehicles without the A- 
pillar and with a curved windshield, which would give a clear field of vision to drivers without causing any hindrance.

Bringing about changes to road infrastructure worldwide ( $>21$ million $\mathrm{km})$ [10], to improve road safety would be capital intensive and feasible only in small regions of LMIC. Thus, addressing the change in the A-pillar design in all future vehicles and retrofitting existing vehicles, in addition to the collective global road safety measures and initiatives, could be a far more effective and viable solution to improve road safety globally. This solution could help meet the United Nations' target of $50 \%$ reduction in road deaths and injuries by 2030. Millions of lives can be saved and injuries prevented, leading to a reduction in economic losses due to road traffic deaths and injuries globally.

Acknowledgements: I especially thank Shrutin Shetty, Innovation and Design Strategy Consultant [Crystal Planet Labs LLP] for constructive discussions.

Declaration of Conflicting Interests: The author declared no potential conflicts of interest with respect to the research, authorship and/or publication of this article.

Funding: The author received no financial support for the research, authorship, and/or publication of this article. The study was carried out on the author's car and expenses for modification were borne by the author, to highlight a concept that may serve a humanitarian need of saving lives from RTC.

\section{References:}

1. World Health Organization - Road traffic injuries - Key facts - 2020. WHO. Geneva

2. WHO. Global status report on road safety 2018. Geneva: World Health Organization, Geneva.

3. World Health Organization. The Global Status Report on Road Safety 2015.

4. World Health Organization and the World Bank. The World report on road traffic injury prevention. 2004 (ISBN: 9241562609)

5. World Bank (2019). Guide for Road Safety Opportunities and Challenges: Low- and Middle-Income Countries Country Profiles. Washington, DC., USA: World Bank.

6. United Nations Road Safety Collaboration, Global Plan for the Decade of Action for Road Safety 2011-2020, World Health Organization, Geneva, 2011

7. World Health Organization. Save LIVES: a road safety technical package WHO (2017)

8. United Nations Road Safety Collaboration, Global Plan for the Decade of Action for Road Safety 2021-2030, World Health Organization, Geneva, 2020.

9. European Commission, Mobility and Transport, Intelligent Transport Systems, Road Mobility and transport. https://ec.europa.eu/transport/themes/its/road_en

10. The World Bank, Data Catalog - GRIP (Global Roads Inventory Project) - 2018 